\title{
Logic Value Fuzzy Subgroup Of a Group
}

\author{
Li Xiao-shen \\ School of Mathematics and Statistics \\ Henan University of Science and Technology \\ Luoyang 471003, China \\ e-mail :hnykli@163.com
}

\author{
Yuan Xue-hai, Wu Le-tao \\ School of Science \\ Dalian University of Technology at Panjin \\ Panjin 124221, China \\ e-mail:yuanxh@dlut.edu.cn
}

\begin{abstract}
The aims of this article are to establish the relationship between the fuzzy algebra and the classical logic and to study fuzzy algebra by the use of classical logic methods. We firstly introduces the concept of the logic value fuzzy subgroup, and studies the relationship between the fuzzy subgroup and its dual. It is pointed out that $H$ is a logic value (normal) fuzzy subgroup of a group $G$ if and only if for all value assignment $v$, the core and the $v$-dual of $H$ is the (normal) subgroup of $G$. Secondly, we study the properties of the logical value fuzzy subgroup, the logical value normal fuzzy subgroup and its quotient groups. Finally, We study the properties of homomorphic image of the logic value fuzzy subgroup. The research of this paper can help to establish the relationship between the fuzzy algebra and the classical logic.
\end{abstract}

Keywords-group; subgroup; normal subgroup; sentence; value assignment; tautology; dual.

\section{INTRODUCTION}

Since A.Rosenfeld introduced the concept of fuzzy subgroup $^{[1]}$, fuzzy algebra has been developed greatly ${ }^{[2]}$. At present, there are three main methods to research fuzzy algebra such as the mthod of membership function of fuzzy set ${ }^{[2,3]}$, the method of the "point - set" neighborhood relation ${ }^{[4,5,6]}$, and the mthod of probability theory ${ }^{[7]}$.

It is well known that the classical algebra theory has the intimate connection with the classical logic ${ }^{[8]}$. The theory of fuzzy subgroups has been studied by using fuzzy logic in $[5,7]$. However, this intimate connection is still not completely understood. This paper attempts to establish a connection between classical propositional logic and fuzzy algebra.

In this paper, we introduce the necessary knowledge in section 2. In section 3 and section 4 , the concept of logical value fuzzy subgroup and the logic value normal fuzzy subgroup are proposed and their properties are studied. In section 5, the properties of homomorphic image of the logic value fuzzy subgroups. In section 6 , we introduce the concept of the strong dual $H^{*}$ and the $v$-dual $H_{v}^{*}$ of logic value fuzzy subgroup $H$ and describe the logic value fuzzy subgroup by strong dual and $v$-dual .

\section{PRELIMINARY}

Let $S$ be the set of sentential variable in propositional logic system $L^{P} . F(S)$ is the formula set in propositional logic $L^{P}$ and $\neg, \wedge, \vee, \rightarrow, \leftrightarrow$ is the connective in $L^{P}$. A value assignment is a mapping $v: S \rightarrow\{0,1\}$. Let $A \in F(S)$ be a formula, if for all value assignment $v$ we get $v(A)=1$, then we call $A$ is a tautology, which is remarked as $\vDash A$ or $\vdash A$.

When $A, B \in F(S)$ and for all value assignment $v$, $v(A)=v(B)$, then we denote $A=B$.

Obviously, $v(A \rightarrow B)=1$ if and only if $v(A) \leq v(B)$.

\section{LOGIC VALUE FUZZY SUBGROUP}

Let $G$ be a group and $F(S)$ be the formula set in propositional logic $L^{P}$.

Definiton 1. Let $H: G \rightarrow F(S)$ be a mapping, if $H$ satisfies:

(1) $\vdash H(e)$,

(2) $\forall x, y \in G, \quad \vdash H(x) \wedge H(y) \rightarrow H(x y)$,

(3) $\forall x \in G, \quad \vdash H(x) \rightarrow H\left(x^{-1}\right)$.

Then we call $H$ a logic value fuzzy subgroup of $G$.

The following conclusions are obvious.

Theorem 1. The mapping $H: G \rightarrow F(S)$ is a logic value fuzzy subgroup of $G$ if and only if for all value assignment $v: S \rightarrow\{0,1\}$, we have

(i) $v(H(e))=1$

(ii) $v(H(x, y)) \geq \min \{v(H(x)), v(H(y))\}, \forall x, y \in G$

(iii) $v\left(H\left(x^{-1}\right)\right) \geq v(H(x)), \forall x \in G$.

Theorem 2. Let $H: G \rightarrow F(S)$ be a mapping,then $H$ is a logic value fuzzy subgroup of $G$ if and only if for all value assignment $v: S \rightarrow\{0,1\}$,

$$
H_{v}=\{x \in G \mid v(H(x))=1\}
$$

is the subgroup of $G, H_{v}$ is called the $v$-core of $H$.

Proof: Let $H$ be a logic value fuzzy subgroup of $G$ and $v: S \rightarrow\{0,1\}$ be a value assignment. Then

(i) $e \in H_{v}$,

(ii) Let $x, y \in H_{v}$, then

$v(H(x y)) \geq \min \{v(H(x)), v(H(y))\}=1$,

and consequently $x y \in H_{v}$.

(iii) Let $x \in H_{v}$, then $v\left(H\left(x^{-1}\right)\right) \geq v(H(x))=1$, and consequently $x^{-1} \in H_{v}$. 
Therefore $H_{v}$ is the subgroup of $G$.

Conversely, Let $v: S \rightarrow\{0,1\}$ be a value assignment, $x, y \in G$. Let $\min \{v(H(x)), v(H(y))\}=1$, then $v(H(x))=v(H(y))=1$. Thus we get $x \in H_{v}, y \in H_{v}$ and $x y \in H_{v}$, so $v(H(x y))=1$, that is

$$
v(H(x y)) \geq \min \{v(H(x)), v(H(y))\} .
$$

Similarly, $v\left(H\left(x^{-1}\right)\right) \geq v(H(x))$.

According to the theorem 1, we know that $H$ is a logic value fuzzy subgroup of $G$.

Theorem 3. Let $H_{1}, H_{2}$ be the logic value fuzzy subgroups of $G$, we define

$$
\left(H_{1} \cap H_{2}\right)(x) \triangleq\left(H_{1}\right)(x) \wedge\left(H_{2}\right)(x),
$$

Then $H_{1} \cap H_{2}$ is the logic value fuzzy subgroup of $G$.

Proof : Let $v$ be a value assignment, then

$\left(H_{1} \cap H_{2}\right)=\left\{x \in G \mid v\left(\left(H_{1} \cap H_{2}\right)(x)\right)=1\right\}=\left\{x \in G \mid v\left(H_{1}(x) \wedge H_{2}(x)\right)=1\right\}$ $=\left\{x \mid v\left(H_{1}(x)\right)=1\right\} \cap\left\{\mid v\left(H_{2}(x)\right)=1\right\}=\left(H_{1}\right)_{v} \cap\left(H_{2}\right)_{v}$

Since $\left(H_{1}\right)_{v},\left(H_{2}\right)_{v}$ are the subgroups of $G$, so $\left(H_{1} \cap H_{2}\right)_{v}$ is a subgroup of $G$. According to theorem 2, $H_{1} \cap H_{2}$ is a logic value fuzzy subgroup of $G$.

\section{LOGIC VALUE NORMAL FUZZY SUBGROUP}

Definition 2. Let $N: G \rightarrow F(S)$ be a logic value fuzzy subgroup of $G$, if

$$
\vdash N(x) \rightarrow N\left(y^{-1} x y\right), \forall x, y \in G,
$$

Then $N$ is called a logic value normal fuzzy subgroup of $G$.

Theorem 4. Let $N: G \rightarrow F(S)$ be a logic value fuzzy subgroup of $G$,if

$\vdash N(x) \rightarrow N\left(y^{-1} x y\right), \forall x, y \in G$,

Then the following conclusions are equivalent:

(1) $N$ is a logic value normal fuzzy subgroup of $G$.

(2) For all value assignment, $v: S \rightarrow\{0,1\}$,we have $v\left(N\left(y^{-1} x y\right)\right) \geq v(N(x))$.

(3) For all value assignment, $v: S \rightarrow\{0,1\}$, we have $v(N(x y))=v(N(y x))$.

(4) For all value assignment, $v: S \rightarrow\{0,1\}, v((x N)(y))=v((N x)(y))$, where $x N, N x: G \rightarrow F(S)$ satisfy

$$
(x N)(y)=N\left(x^{-1} y\right),(x N)(y)=N\left(y x^{-1}\right) .
$$

Proof: (1) $\Rightarrow$ (2) Let $v: S \rightarrow\{0,1\}$ be a value assignment, by definition 1 , we have

$$
\begin{aligned}
& v\left(N(x) \rightarrow N\left(y^{-1} x y\right)\right)=1 \Rightarrow v\left(N\left(y^{-1} x y\right)\right) \geq v(N(x)) . \\
& (2) \Rightarrow(3) \quad v(N(x y))=v\left(N\left(y^{-1} y x y\right)\right) \geq v(N(y x)),
\end{aligned}
$$
similarly, $v(N(y x)) \geq v(N(x y))$, so $v(N(x y))=v(N(y x))$.

(3) $\Rightarrow$ (4)

$$
v((x N)(y))=v\left(N\left(x^{-1} y\right)\right)=v\left(N\left(y x^{-1}\right)\right)=v((N x)(y)) .
$$

The following conclusions are obvious.

Theorem 5. The mapping $N: G \rightarrow F(S)$ is a logic value normal fuzzy subgroup of $G$ if and only if for all value assignment $v: S \rightarrow\{0,1\}, H_{v}=\{x \mid v(N(x))=1\}$ is a normal subgroup of $G$.

Definition 3. Let $A, B$ be the mapping from $G$ to $F(S)$ and $\{A(x) \mid x \in G\}$ and $\{B(x) \mid x \in G\}$ are finite sets, we define

$$
(A B)(y)=\bigvee_{x \in G}\left(A\left(y x^{-1}\right) \wedge B(x)\right),
$$

$A B$ is called the product of $A$ and $B$.

Theorem 6. (1) If $N_{1}, N_{2}$ are logic value normal fuzzy subgroups of $G$, then $N_{1} \cap N_{2}$ is a logic value normal fuzzy subgroup of $G$.

(2) If $N$ is a logic value normal fuzzy subgroup of $G, H$ is a logic value normal fuzzy subgroup of $G$ and $\{N(x) \mid x \in G\}$ and $\{H(x) \mid x \in G\}$ are finite sets, then $N G$ is a logic value normal fuzzy subgroup of $G$.

Proof : As

$$
(N H)(y)=\bigvee_{x \in G}\left(N\left(y x^{-1}\right) \wedge H(x)\right),
$$

we get

$$
\begin{aligned}
y \in(N H)_{v} \Leftrightarrow & \exists x \in G, v\left(N\left(y x^{-1}\right)\right)=v((H x))=1 \\
& \Leftrightarrow \exists x \in G, x \in H_{v}, y x^{-1} \in H_{v} . \\
& \Leftrightarrow y \in N_{v} H_{v} .
\end{aligned}
$$

As $N_{v}$ is a normal subgroup of $G$ and $H_{v}$ is a subgroup of $G$, so $N_{v} H_{v}$ is a subgroup of $G$. Thus $(N H)_{v}=N_{v} H_{v}$ is the subgroup of $G$.

Therefore $N H$ is a logic value fuzzy subgroup of $G$.

Theorem 7. Let $N$ be a logic value normal fuzzy subgroup of $G$ and $\{N(x) \mid x \in G\}$ is a finite set, let $G / N=\{x N \mid x \in G\}$, then (1) $G / N$ can form a group; (2) For all value assignment $v, G / N_{v}$ is isomorphic to $G / N$.

Proof : (1) Let $x N, y N \in G / N$, we prove

$$
(x N)(y N)=x y N \text {, }
$$

that is for all value assignment $v$, we have 
In fact, $v((x N)(y N)(z))=1 \Leftrightarrow \exists a \in G$ such that $v\left(\left((x N)\left(z a^{-1}\right)\right) \wedge\left((y N)\left(a^{-1}\right)\right)\right)=1$

$\Leftrightarrow \exists a \in G, v\left(N\left(x^{-1} z a^{-1}\right) \wedge N\left(y^{-1} a\right)\right)=1$

$\Leftrightarrow \exists a \in G, x^{-1} z a^{-1} \in N_{v}, y^{-1} a \in N_{v}$

$\Leftrightarrow y^{-1} a \in N_{v}, a^{-1}\left(x^{-1} z\right)=a^{-1}\left(x^{-1} z a^{-1}\right) a \in N_{v}$

$\Leftrightarrow(x y)^{-1} z=y^{-1} a \cdot a^{-1} x^{-1} z \in N_{v}$

$\Leftrightarrow v\left(N\left((x y)^{-1}(z)\right)\right)=1 \Leftrightarrow v((x y N)(z))=1$

Therefore, $v((x N)(y N)(z))=v((x y N)(z))$, that is $(x N)(y N)=(x y) N$. Hence $G / N$ forms a group.

(2) Let $\phi: G / N_{v} \rightarrow G / N, x N_{v} \mapsto x N$, then

$$
\begin{aligned}
& x N_{v}=y N_{v} \Leftrightarrow y^{-1} x \in N_{v} \\
& \Leftrightarrow v\left(N\left(y^{-1} x\right)\right)=1 \Leftrightarrow v\left(N\left(x^{-1} y\right)\right)=1
\end{aligned}
$$

So we get

$$
v((x N)(z))=1 \Leftrightarrow v\left(N\left(x^{-1} z\right)\right)=1 \Leftrightarrow v\left(N\left(x^{-1} y \cdot y^{-1} z\right)\right)=1
$$

Then

$$
\begin{aligned}
v(x N)(z)) & \geq \min \left\{v\left(N\left(x^{-1} y\right)\right), v\left(N\left(y^{-1} z\right)\right)\right\} \\
& \left.=v\left(N\left(y^{-1} z\right)\right)=v(y N)(z)\right)
\end{aligned}
$$

Similarly, $v((y N)(z)) \geq v((x N)(z))$.

Thus $v((y N)(z))=v((x N)(z))$ and consequently $y N=x N$.

Therefore,$\phi$ is a mapping and $\phi$ is a surjection.

Let $\phi\left(x N_{v}\right)=\phi\left(y N_{v}\right)$, thus $x N=y N$. Then for all value assignment $v$ and $z \in G$, we have $v((y N)(z))=v((x N)(z))$, that is $v\left(N\left(x^{-1} z\right)\right)=v\left(N\left(y^{-1} z\right)\right), \forall z \in G$, thus $v\left(N\left(x^{-1} y\right)\right)=v\left(N\left(y^{-1} y\right)\right)=v(N(e))=1$, then $x^{-1} y \in N_{v}$, we get $x N_{v}=y N_{v}$.

Therefore, $\phi$ is a isomorphism.

So $G / N_{v}$ is isomorphic to $G / N$.

V. THE PROPERTY OF HOMOMORPHISM IMAGE ON THE LOGIC VALUE FUZZY SUBGROUP

Definition 4. Let $f: G_{1} \rightarrow G_{2}$ be a surjective homomorphism of group,

$$
H: G_{1} \rightarrow F(S), K: G_{2} \rightarrow F(S)
$$

be a mapping and $\{H(x) \mid x \in G\}$ be a finite set, then

$$
f(H)(y) \triangleq \underset{f(x)=y}{\vee} H(x), f^{-1}(K)(x) \triangleq K(f(x)) .
$$

Theorem 8. Let $f: G_{1} \rightarrow G_{2}$ be surjective homomorphism of group. Then we have

(1) If $H$ is a logic value (normal) fuzzy subgroup of $G_{1}$ and $\{H(x) \mid x \in G\}$ is a finite set, then $f(H)$ is a logic value (normal) fuzzy subgroup of $G_{2}$.

(2) If $K$ is a logic value (normal) fuzzy subgroup of $G_{1}$, then $f^{-1}(K)$ is a logic value (normal) fuzzy subgroup of $G_{1}$.

Proof : (1) Let $H$ be a logic value (normal) fuzzy subgroup of $G_{1}, v$ be a value assignment and $y \in(f(H))_{v}$, then $v(f(H)(y))=1 \Leftrightarrow \exists x \in G, f(x)=y$ and $v(H(x))=1$

$\Leftrightarrow \exists x \in H_{v}$ such that $f(x)=y \Leftrightarrow y \in f\left(H_{v}\right)$,

Therefore, $(f(H))_{v}=f\left(H_{v}\right)$.

Because $H_{v}$ is the subgroup of $G_{1}, f\left(H_{v}\right)$ is the subgroup of $G_{2}$.

Therefore, $f(H)$ is a logic value fuzzy subgroup of $G_{2}$, when $H$ is a logic value normal fuzzy subgroup of $G_{1}$,we can similarly prove that $f(H)$ is a logic value (normal) fuzzy subgroup of $G_{2}$.

(2) $x \in\left(f^{-1}(K)\right)_{v} \Leftrightarrow v\left(f^{-1}(K)(x)\right)=1$ $\Leftrightarrow v(K(f(x)))=1 \Leftrightarrow f(x) \in K_{v} \Leftrightarrow x \in f^{-1}\left(K_{v}\right)$,

Thus $\left(f^{-1}(K)\right)_{v}=f^{-1}\left(K_{v}\right)$. Therefore when $K$ is the logic value (normal) fuzzy subgroup of $G_{2}, f^{-1}(K)$ is the logic value (normal) fuzzy subgroup of $G_{1}$.

\section{THE DUAL OF LOGIC VALUE FUZZY SUBGROUP}

Definition 5. Let $H: G \rightarrow F(S)$ be a mapping, $v: S \rightarrow\{0,1\}$ is a value assignment, $A \in F(S)$, let

$$
\begin{aligned}
& H^{*}(A)=\{x \mid \vdash A \rightarrow H(x)\}, \\
& H_{v}^{*}(A)=\{x \mid v(A) \leq v(H(x))\}
\end{aligned}
$$

$H^{*}$ is called the strong dual of $H$ and $H_{v}^{*}$ is called the $v$-dual of $H$.

Theorem 9. (1) $H$ is a logic value (normal) fuzzy subgroup of $G$ if and only if for all $A \in F(S), H^{*}(A)$ is a (normal) subgroup of $G$. (2) $H$ is a logic value (normal) fuzzy subgroup of $G$ if and only if for all $A \in F(S)$ and value assignment $v, H_{v}^{*}(A)$ is a (normal) subgroup of $G$.

Proof :Let $H$ be a logic value (normal) fuzzy subgroup of $G, A \in F(S)$, as $H(e)$ is a tautology ,so $H^{*}(A) \neq \varnothing$.

Let $x, y \in H^{*}(A)$, then for all value assignment $v$, we have

$$
v(A \rightarrow H(x))=v(A \rightarrow H(y))=1,
$$

Then

$$
v(A) \leq \min \{v(H(x)), v(H(y))\} \leq v(H(x y)),
$$

Thus $\quad v(A \rightarrow H(x y))=1$, It follows that $\vdash A \rightarrow H(x y)$ and consequently $x y \in H^{*}(A)$.

Similarly we can prove $x^{-1} \in H^{*}(A)$ from $x \in H^{*}(A)$. 
Therefore $H^{*}(A)$ is the subgroup of $G$.

When $H$ is a logic value normal fuzzy subgroup of $G$, similarly we can prove $H^{*}(A)$ is the normal subgroup of $G$.

(1) $" \Leftarrow "$ Let $A=H(x) \wedge H(y)$, as $H^{*}(A)$ is the normal subgroup of $G$ and $x \in H^{*}(A), y \in H^{*}(A)$, so $x y \in H^{*}(A)$, we have that $\vdash A \rightarrow H(x y)$, that is

$\vdash H(x) \wedge H(y) \rightarrow H(x y)$.

Let $A=H(x)$, thus $H^{*}(A)$ is the subgroup of $G$ and $x \in H^{*}(A)$, then $x^{-1} \in H^{*}(A)$. We have that

$$
\vdash H(x) \rightarrow H\left(x^{-1}\right) .
$$

Let $A$ be a tautology, according to $H^{*}(A)$ is the subgroup of $G$, we know $e \in H^{*}(A)$, then $\vdash A \rightarrow H(e)$, thus $\vdash H(e) . H$ is a logic value fuzzy subgroup of $G$.

Similarly, for all $A \in F(S), H^{*}(A)$ is a normal subgroup of $G$. therefore $H$ is the logic value normal fuzzy subgroup of $G$.

(2) $" \Leftarrow$ "Because $H$ is the logic value fuzzy subgroup of $G$,we get $\vdash H(e)$. Therefore for all formula $A \in F(S)$, there is $e \in H_{v}^{*}(A)$.

Let $x, y \in H_{v}^{*}(A)$, which results in

$$
v(A) \leq v(H(x)), v(A) \leq v(H(y)) .
$$

Thus $v(A) \leq \min \{v(H(x)), v(H(y))\} \leq v(H(x y))$, then $x y \in H_{v}^{*}(A)$.

Let $x \in H_{v}^{*}(A)$, thus $v(A) \leq v(H(x)) \leq v\left(H\left(x^{-1}\right)\right)$, then $x^{-1} \in H_{v}^{*}(A)$, therefore $H_{v}^{*}(A)$ is a subgroup of $G$.

Similarly, we can prove that $H^{*}(A)$ is the subgroup of $G$ when $H$ is a logic value normal fuzzy subgroup of $G$ for all formula $A$.

" $\Leftarrow$ "let $v(A)=1, x, y \in H_{v}$, hence $v(H(x))=v(H(y))=1$. So we have $v(A) \leq v(H(x)), v(A) \leq v(H(y))$, that is $x, y \in H_{v}^{*}(A)$, thus $x y \in H_{v}^{*}(A)$, then $v(A) \leq v(H(x y))$. We have $v(H(x y))=1$. Hence $H_{v}$ is the subgroup of $G$.

Therefore $H$ is a logic value normal fuzzy subgroup of $G$.

The others are similar.

We are able to get the following conclusions obviously.

\section{Theorem 10.}

(1) $\left(H_{1} \cap H_{2}\right)^{*}(A)=H_{1}{ }^{*}(A) \cap H_{2}{ }^{*}(A)$

(2) $\left(H_{1} \cap H_{2}\right)_{v}^{*}(A)=\left(H_{1}\right)_{v}^{*}(A) \cap\left(H_{2}\right)_{v}^{*}(A)$

(3) $H^{*}(A \vee B)=H^{*}(A) \cap H^{*}(B)$
(4) $H_{v}{ }^{*}(A \vee B)=H_{v}^{*}(A) \cap H_{v}^{*}(B)$

(5) $H^{*}(A \wedge B) \supseteq H^{*}(A) \cup H^{*}(B)$

(6) $H_{v}^{*}(A \wedge B)=H_{v}^{*}(A) \cup H_{v}^{*}(B)$

(7) $H_{v}^{*}(A)=\left\{\begin{array}{l}G, \quad v(A)=0 \\ H_{v}, v(A)=1\end{array}\right.$

(8) $H^{*}(A)=\left\{\begin{array}{l}\{x \mid \vdash H(x)\}, A \text { is a tautology } \\ G, \quad A \text { is a contradictory }\end{array}\right.$

(9) $H^{*}(A) \cap H^{*}(\neg A)=\{x \mid \vdash H(x)\}$

\section{CONCLUSION}

This article presents the concepts of logic value fuzzy subgroup, the core $H_{v}$, the strong dual $H^{*}$ and the $v$-dual $H_{v}^{*}$ of logic value fuzzy subgroup. It is pointed out that we are able to describe the logic value fuzzy subgroup $H$ of $G$ by use of $H_{v}, H^{*}, H_{v}^{*}$. The research of this paper can be seen that the existing fuzzy algebra theory is easy to generalize to the theory of logic value fuzzy algebra.

\section{REFERENCES}

[1] A.Rosenfeld, "Fuzzy groups", Journal of Mathematical Analysis and Applications, 35(3), pp.512-517.1971.

[2] J.N.Mordeson , D.S.Malik, Fuzzy Commutative Algebra, Singapore: World Scientific Publishing, 1998.

[3] X.H.Yuan, E.S.Lee. "Fuzzy group based on fuzzy binary operation", Computers \& Mathematics with Applications, 47, pp. 631-641,2004.

[4] S.K.Bhakat, P.Das ," On the definition of a fuzzy subgroup", Fuzzy Sets and Systems, 51(2), pp.235-241,1992.

[5] X.H.Yuan, C.Zhang, Y.H.Ren , "Generalized fuzzy groups and manyvalued implications", Fuzzy Sets and Systems, 138, pp.205-211,2003.

[6] X.H.Yuan, E.S.Lee, "A fuzzy algebraic system based on the theory of falling shadows", Journal of Mathematical Analysis and Applications, 208, pp.243-251,1997.

[7] X.H.Yuan, H.X.Li, E.S. Lee, "On the definition of intuitionistic fuzzy subgroups", Computer and Mathematics with Applications, 59(9), pp. 3117-3129, 2010.

[8] D.W.Bames,J.M.Mark, An Algebraic Introduction to Mathematical Logic, New York :Springer, 1975. 